\title{
Osteonecrosis of the jaw related to non-antiresorptive medications: A systematic review
}

\author{
O. Nicolatou-Galitis ${ }^{1}$, M. Kouri ${ }^{1}$, E. Papadopoulou ${ }^{1}$, E. Vardas ${ }^{1}$, D. Galiti ${ }^{1}$, J.B.Epstein ${ }^{2}$, S. Elad ${ }^{2}$, G. \\ Campisi $^{3}$, N. Tsoukalas ${ }^{1}$, K. Bektas-Kayhan ${ }^{4}$, W. Tan², JJ Body ${ }^{5}$, C. Migliorati², R. Lalla², for the \\ MASCC Bone Study Group. \\ ${ }^{1}$ Greece, ${ }^{2}$ USA, ${ }^{3}$ taly, ${ }^{4}$ Turkey, ${ }^{5}$ Belgium
}

\section{Objective}

To review characteristics of ONJ in cancer patients receiving medications without known antiresorptive properties, such as antiangiogenics, tyrosine kinase inhibitors, mammalian target of rapamycin inhibitors, immune checkpoint inhibitors and cytotoxic chemotherapy.

\section{Methods}

A systematic review of the literature between 2009-2017 was conducted by the BSG.

\section{Results}

Of 6,249 articles reviewed and from personal communication, 42 ONJ cases related to non-antiresorptives were identified.

Medications included: bevacizumab, aflibercept, sunitinib, imatinib, cabozantib, sorafenib, regorafenib, axitinib, pazopanib, dasatinib, everolimus, temsirolimus, rituximab, BRAFis (dabrafenib \& trametinib), and the immune check point inhibitors (ipilimumab \& nivolumab).

Cytotoxic medications: (1) cytarabine, idarubicin and daunorubicn, (2) gemcitabine, vinorelbine, and doxorubicin, (3) doxorubicin and cyclophosphamide, and (4) 5-azactytidine as monotherapy.

Similarities and differences were noted when compared to ONJ related to BTAs.

Similarities: Males and females, middle aged, were equally affected, ONJ stage 2 was most common, posterior mandible was often affected, exposed bone [FIGURE 1], pain and infectious manifestations were common at diagnosis. Radiology [FIGURE 2], histology and management were also similar to that of ONJ related to BTAs, with medication often discontinued. Delayed diagnosis (median 8 weeks) was noted.

Differences: Earlier time to ONJ onset (median 20 weeks), absence of trigger event (40\%), greater likelihood of healing and shorter healing time (median 8 weeks). Gastrointestinal and renal cell carcinomas predominated reflecting the different medications. No patients with prostate cancer were found.

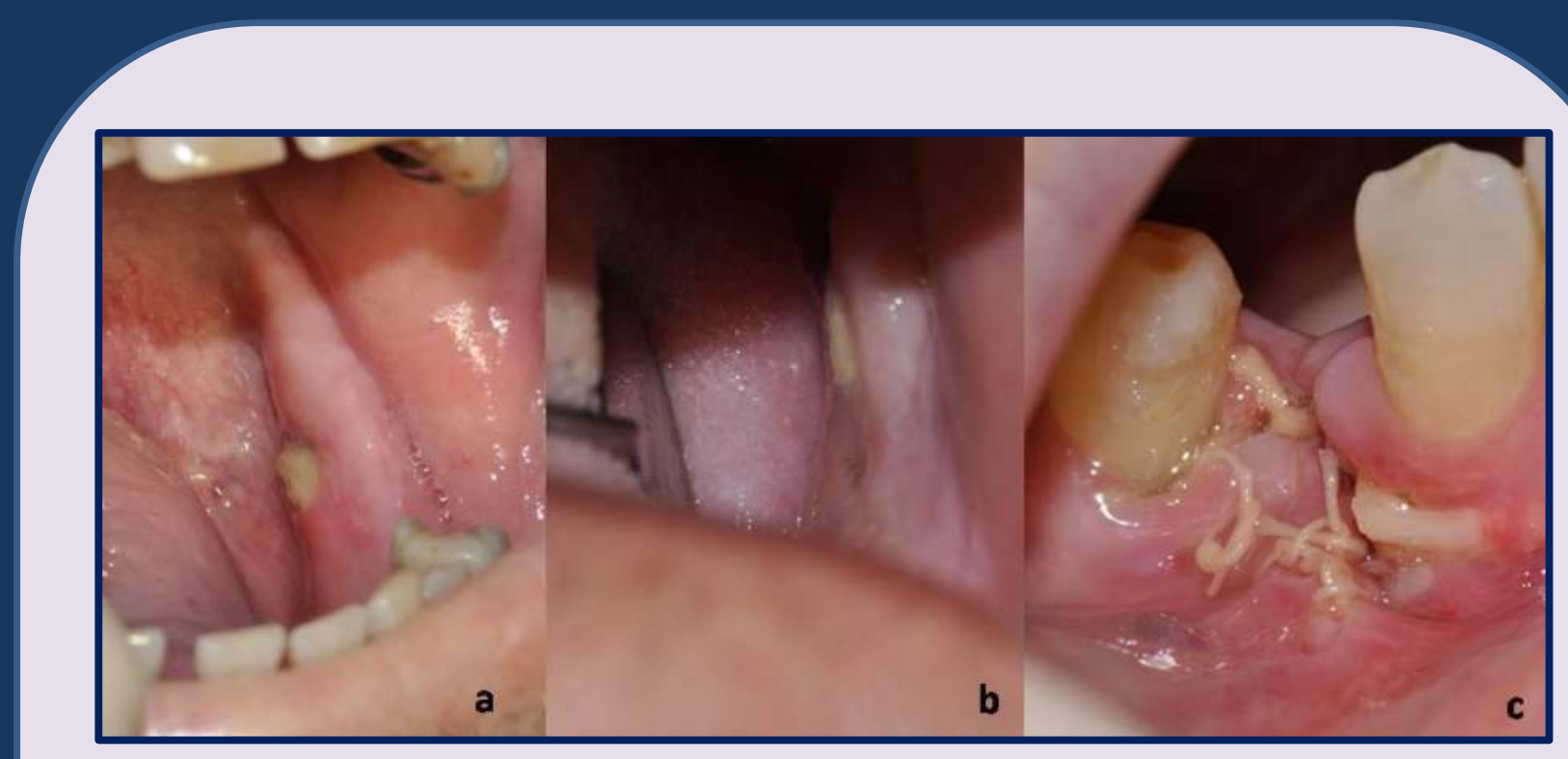

FIGURE 1. a. Necrotic, exposed bone (ONJ) is observed on the left posterior lingual mandible. Patient was managed for lung cancer with dabrafenib and trametinib. $\mathbf{b}$. ONJ is observed on the left lingual mandible. The patient was managed for lung cancer with nivolumab. c. ONJ extends on the buccal aspect, right mandible, after the dental extraction of the 1st premolar. Exposed bone was observed prior to dental extraction by the author, who provided this case. The patient was managed for gastrointestinal stromal tumor with imatinib.

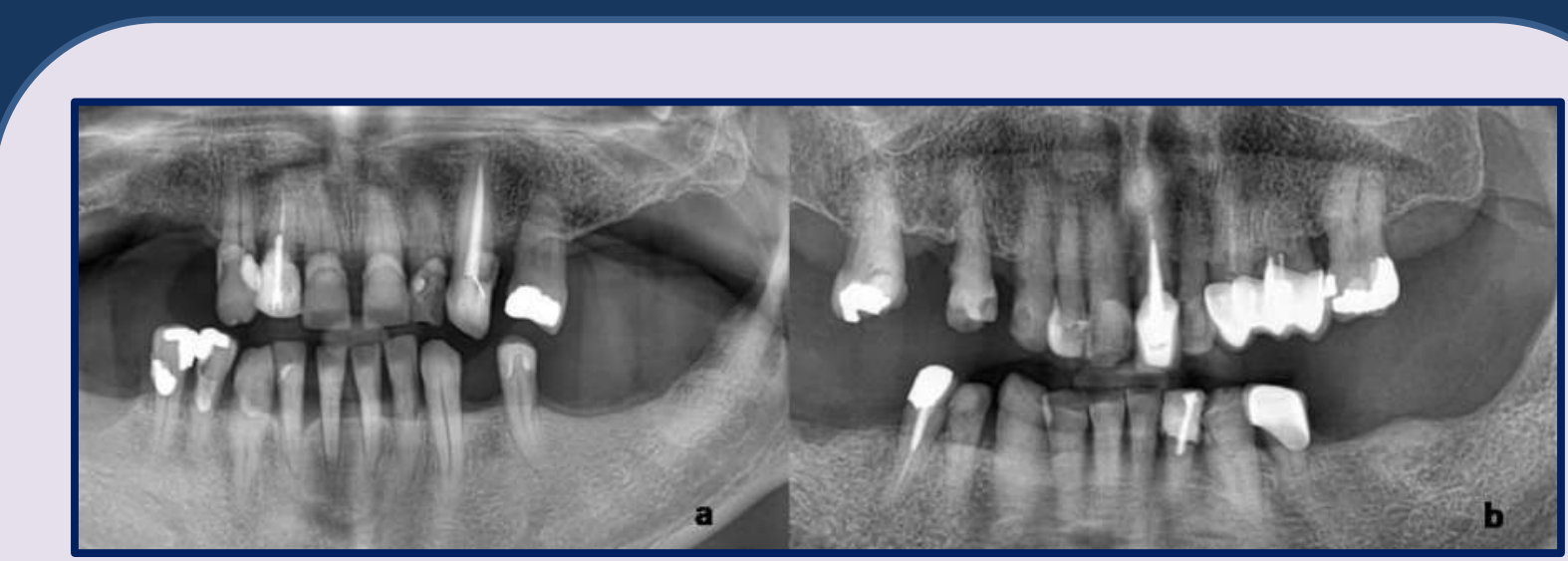

FIGURE 2. a. Orthopantomograph shows osteolysis on the left mandible of the patient from Figure $1 \mathrm{a}$ at the area of the clinical necrotic, exposed bone. $\mathbf{b}$. Orthopantomograph osteolysis on the left mandible of the patient from Figure $1 b$ at the area of clinical necrotic exposed bone.

\section{Conclusions}

ONJ related to non-antiresorptive medications may have better prognosis compared to the BTA-related, suggested by greater likelihood of healing and shorter healing time. However, the delay in diagnosis highlights the need for more education. This is the first attempt to characterize ONJ associated with different nonantiresorptives, including BRAF and immune check point inhibitors.

\section{Acknowledgement}

We thank the MASCC Board for the opportunity to work on this systematic review, within the Bone Study Group. 